\title{
Research on the multi energy complementation of wind power consumption
}

\author{
Jingfeng Zheng ${ }^{1}$ \\ ${ }^{1}$ School of Information and Control \& \\ Nanjing University of Information Science and Technology,Nanjing 210044,China
}

\begin{abstract}
:
Multi energy complementation has gradually become a research hotspot in the future power system. For the whole multi energy complementary system, its fundamental purpose is to consider the stability of the overall power output. In this paper, the composition of multi energy complementary energy and the form of multi energy complementary function are analyzed, and the minimum mathematical model of the whole power fluctuation is established. The particle swarm optimization (PSO) is used to carry out a simulation study in a certain area of Xinjiang. It is concluded that the multi energy complementation is an effective means to improve the quality of wind power distribution and to solve the problem of abandoning the wind. Through the combination of clean energy forms such as solar energy and hydropower, the use value of new energy is fully developed, and the use of traditional fossil energy is reduced, and the wind power consumption has a positive effect.
\end{abstract}

Keywords: Multi energy complementation; Stationarity; PSO; wind power consumption ; clean energy

\section{I . Introduction}

With the depletion of fossil energy on the earth, domestic and international attention is generally paid to the utilization of renewable energy. The integration of high proportion of renewable energy has become the development direction of power systems, while the volatility and randomness of wind power generation and solar power generation are large, it has a greater impact to the safe and stable operation of power Systems. Hydropower is an adjustable, large-scale clean energy source that can effectively stabilize wind power and photovoltaic power generation fluctuations and improve the capacity of the grid to absorb it.

Multi energy complementation is a new, comprehensive energy dispatch mechanism. The multi energy complementary mechanism is to use other forms of power input ports to compensate for the variability, volatility and randomness of wind power output. Under the multi energy complementary energy dispatching mechanism, the supply side simultaneously parallels multiple energy supply modes, and according to the current situation of the entire energy transmission network, rationally schedules the input and distribution of each energy supply mode, or stores and conversion some of the supplied electrical energy,in order to compensate for the shortcomings in the transmission of electrical energy in a single energy form. Multi energy complementation is a comprehensive and complementary utilization at the system level according to various energy sources. It is a coordinated scheduling of multiple energy 
sources, and reasonable allocation of various energy conversions and transmissions. It is not a superposition of simple multiple energy supply methods.

Most of the current research is a single power generation system $^{[1-3]}$, and there are few studies on multi energy complementary systems.For the whole pluripotent complementary system, the fundamental purpose is to consider the stability of the overall power output. This paper analyzes the composition of multi energy complementary energy and the multi energy complementary mode of action, and establishes the minimum mathematical model of the overall power fluctuation. Using the particle swarm optimization algorithm to simulate a certain place in Xinjiang, it is concluded that multi energy complementation is an effective means to improve the quality of wind power distribution and solve the problem of abandoned wind. Through the cooperation with clean energy forms such as solar energy and hydropower, the utilization value of new energy sources has been fully developed, and the use of traditional fossil energy sources has been reduced, and the promotion of wind power consumption has played a positive role.

\section{II . Multi energy complementation design}

\section{A. Solar energy generation}

Solar energy is an inexhaustible source of energy in a sunny place, and it is a clean and green new energy source. Solar energy does not emit greenhouse gases and other harmful gases, and it does not cause environmental pollution. In recent years, the cost of generating electricity for solar energy has been declining. However, solar power generation has the characteristics of volatility and is seriously affected by weather factors. Therefore, the construction of large-scale solar power generation facilities has high requirements for site selection, and it is necessary to have sufficient light for a long time in a year. Solar power generation is variability, and day and night replacement determines that solar power generation is a long-term intermittent production.
Although the electricity consumption at night will be relatively low, solar power generation is completely inoperative at night, which determines that it is impossible to supply power in a separate power supply. Other forms of energy supply are required for compensation.

\section{B. Wind power}

Wind energy is a new type of clean green energy. In general, wind energy is geographically distributed more evenly and more abundantly than solar energy. Although the construction of wind power generation requires relatively large surface area and space, its construction cost is relatively low, and environmental pollution is basically not generated in the process of wind power generation. Wind power generates electricity in the same way as solar energy, but also has volatility and variability. The power generated by wind energy depends on the size and direction of the wind. Although it is basically possible to classify the average wind energy density line on the surface, this is only a statistical average. The magnitude and direction of the wind changes at different times during the different periods of the year, so the power generated by the power will also change. Compared to solar energy, this fluctuation is more frequent and occurs in real time within twenty-four hours a day, so other forms of energy supply are also required to compensate.

\section{Thermal power generation}

Thermal power generation is the main form of power generation in China at present, so a part of the multi energy complementary system will definitely include thermal power generation.

Thermal power generation should reasonably limit its use in multi energy complementary systems because of the non-renewability of fossil energy and the pollution of thermal power generation. Thermal power generation can participate in a certain degree of peak shaving control, but the output adjustment speed of thermal power generation is relatively slow, and it needs to be 
controlled in combination with the power generation situation of wind power and solar power generation.

\section{Hydroelectric power}

Water power generation is earlier than wind energy and solar power generation, and water power generation basically does not emit environmental pollutants. The advantage of hydroelectric power generation is that there will be no large-scale fluctuations in power generation in a short period of time, which is much better than solar and wind energy.

The disadvantage of hydropower is that the selection of addresses is very strict and the applicable area is particularly narrow. If the impact on the surrounding environment is taken into consideration, and the nodes suitable for hydropower generation in the river are selected, such site selection is very rare. And the construction requirements of hydropower generation are relatively high, and relatively advanced construction techniques are needed. Unsafe water conservancy facilities are a great hidden danger. Hydropower has a certain seasonality. Generally, rivers have a period of abundant water and dry season. The power of hydropower in these two periods will be different. This can be adjusted by the reservoir.

Since the unit of the hydropower facility has a relatively fast response speed, it can be combined with wind power and solar power to adjust the total power generation.

\section{E. Pumped storage hydropower station}

The pumped storage power station is a special type of hydropower station. The principle of power generation is similar to that of a hydropower station. It also uses the potential energy of water to drive turbines between waterways ${ }^{[10]}$. Compared with the hydropower station, the difference is that the hydropower station relies on the original river water, and builds the dam to take the high water drop in the place with large ground potential to generate electricity. Pumped storage also has a dam and a higher-lying reservoir, but the water in the reservoir is pumped up by other means of electricity. At the time of power generation, water is discharged through the reservoir to convert the potential energy of the water into electrical energy.

Pumped storage power plants can serve as the primary conditioning port in a multi energy complementary system. Because it is not a purely power generation unit, it is a unit that can convert energy into each other and temporarily store electrical energy. Especially for wind power, it can be optimized by simply using the technology of pumped storage. While the wind turbine is running, the air compressor can be used to store excess energy by storing energy such as compressed air.

Pumped-storage hydropower stations are responsible for the main peak-shaving tasks and rapid response in the multi-energy complementary system, and play an important role in the phase modulation and frequency modulation of the system. The rational introduction of pumped storage facilities can effectively improve the ability of the entire pluripotent complementary system to accept wind power and solar power generation .

\section{Multi energy complementary form of action}

\section{A. Wind power and solar power complement each other}

Wind power generation and solar power generation are two forms of power generation and new energy generation has randomness and volatility. There is no clear complementary relationship between power generation and power generation. However, if there are specific geographical factors, the power generation and output of the two can be complemented. For example, in some areas with large temperature difference between day and night, there is windy nighttime and plenty of daylight. In this way, solar 
power generation during the day and night wind power output can complement the power generation between the two. At the same time, in the appropriate areas to build wind power and solar power projects, the two can use the same transmission line for transmission, which can save a certain transmission line construction costs. At the same time, the complementary output of these two new energy sources can improve the power transmission efficiency of the line, and it has higher stability as a unified new energy access node.

\section{B. New energy (wind, light) power generation and hydropower complement each other}

Due to the existence of reservoirs, hydropower facilities can adjust their power generation to a certain extent, which complements the randomness and variability of wind power and solar power. When the electricity load is low, or when the power generation of wind power generation and solar power generation exceeds the average level, the hydropower facility can reduce the power generation by reducing the power generation by closing the gate and storing the water in the reservoir. In wind power facilities and solar power generation facilities, due to environmental and meteorological factors, power generation is reduced below the average level, or when the electricity load is low, hydropower facilities can discharge large amounts of saved water, increase their power generation, and fill the low power generation.

\section{New energy (wind, light) power generation and pumping storage complement each other}

As an electric energy conversion and temporary storage facility, pumped storage power station is a key complementary way for new energy generation with variability and randomness. The complementary role of pumped storage in wind power generation is now more pronounced. The first complementary form of wind power generation and pumped storage is the storage of electrical energy resources for local wind power generation. During the peak period of wind power generation, the wind power generation facility supplies power to the pumped storage hydropower station, and temporarily converts this part of the electric energy into potential energy by pumping water into the reservoir. During the peak hours of electricity consumption, the electric energy is reoutputted and supplied to the power grid by means of water discharge, which suppresses the instability factors of wind power generation, improves the stability of its power output, and enhances the local grid's ability to accept wind power.

The second complementary form of wind power generation and pumped storage is to improve the on-grid transmission of wind power. The operation mode is basically the same as the first complementary form, but the pumped storage power station is not connected to the local power grid, but serves as an input node for electric energy, and the grid is used for power transmission. Due to various instability of wind power, direct access to the power grid for transmission will cause a certain degree of energy loss, and is also detrimental to the safety of the transmission line and the entire power grid. The construction of pumped storage power stations at the output node will control the peak output and valley filling of the wind power generation output, which is more conducive to the wind power generation capacity to be connected to the grid for transportation.

\section{Thermal power generation complements other forms of energy}

The stability of thermal power generation itself does not need to be adjusted. The main purpose of accessing the multi-energy complementary system is to limit its power generation, reduce the consumption of fossil energy and the impact of environmental pollution. Hydropower is a good complement to thermal power generation. Hydropower has a relatively long peak period of power generation, and this peak period generally occurs in the summer. Because of the large-scale use of refrigeration equipment such as air 
conditioners in summer and the peak period of agricultural irrigation, the overall electricity demand will increase significantly. Through the compensation of hydropower, it is not necessary to invest too much thermal power resources, which can reduce the environmental impact and energy consumption of thermal power generation . Wind power and solar power generation have little effect in the peaking of thermal power generation due to their own instability, but they can replace part of the amount of thermal power generation in various forms of energy co-transport. At the peak of wind power generation and solar power generation, the investment in thermal power generation is appropriately reduced, and the power generation capacity of thermal power generation is suppressed while ensuring that the overall transmission efficiency of the transmission line is not affected. However, when wind power generation and solar power generation are reduced to a low level, it is necessary to appropriately increase the power generation input of thermal power generation to ensure the power supply stability of the entire multi-energy complementary system.

\section{Multi-energy complementary model}

\section{A.Mathematical modeling}

For the entire pluripotent complementary system, the fundamental purpose is to reduce the current energy transmission by mobilizing the wind energy generation, solar power generation, hydropower and other clean energy transmission modes without the large fluctuation of the overall power output. The main body, the operation and investment of thermal power generation. The variance can be used to characterize the overall fluctuations of the system. The purpose is to make the difference between the overall output of clean energy and the demand for energy input in the multi-energy complementary system stable, so that a stable amount of thermal energy can be stably input. . So the objective function is:

$$
\min F=\frac{1}{T} \sum_{t=1}^{T}\left(P_{g t}-P_{g t, a v e}\right)^{2}
$$

Where $P_{g t}$ is the difference between the output demand of the system at time $t$ and the output of the clean energy; $P_{g t, a v e}$ is the average of the difference in demand for the multi-energy complementary system at time $t$.

Wind, solar and water are all clean energy, so the difference between the output demand of the system and the output of clean energy and the difference in demand for multi-complementary systems are:

$$
\begin{aligned}
& P_{g t}=P_{l t}-\left(P_{f t}+P_{s t}+P_{y t}\right) \\
& P_{g t, a v e}=\frac{1}{T} \sum_{t=1}^{T} P_{g t}
\end{aligned}
$$

Where $P_{l t}$ is the demand for load at time $\mathrm{t} ; P_{f t}$ is wind energy output; $P_{s t}$ is water energy output; $P_{y t}$ is solar energy output; T is time.

The traditional thermal power generator model is:

$$
W_{H}=\sum_{t=1}^{T} \sum_{i=1}^{N}\left(a_{i} P_{i t}^{2}+c b_{i} P_{i t}+m S_{i t}\right)
$$

Among them, $W_{H}$ is the operating output of thermal power; $a, b$ is the reference coefficient of the output of the cost of the energy consumed by the thermal power unit, and $P_{i t}$ is the output of the energy cost of the operation of the i-th thermal power unit at time t. T; $\mathrm{N}$ is the time label and number of operation of the thermal power unit; 
$S_{i t}$ is the energy consumption of the i-th thermal power unit during the state switching phase at time $\mathrm{t} ; \mathrm{m}$ is the state switching of the thermal power unit in the unit time period The total number of times; $c$ is the control quantity, which is generally 0 , and vice versa.

\section{B.Restrictions}

1) Thermal power unit output constraints

For the thermal power project, the i-th unit, the quantitative representation of its output is: this output has its upper and lower limits:

$$
P_{i \min } \leq P_{i t} \leq P_{i \max }
$$

$P_{i \min }$ and $P_{i \max }$ are the minimum and maximum output of the i-th thermal power unit, respectively.

2) Time and rate constraints for the switching of the thermal power unit

Since the switching of thermal power is slow, it is necessary to consider the time and rate of switching:

$$
D_{i \min } \leq P_{i t}-P_{i(t-1)} \leq D_{i \max }
$$

Among them, $D_{i \min }$ and $D_{i \max }$ are the minimum and maximum capacity of the i-th thermal power unit treatment change respectively, and the output force rises to decrease the positive output force to negative; $P_{i t}-P_{i(t-1)}$ is the output difference between the two time intervals of the ith thermal power unit.

3) The time limit for the opening and closing of the thermal power unit

Since the thermal power unit also has the problem of state switching, that is, the opening and closing of the unit are also time-required under normal circumstances, and cannot be switched multiple times too quickly:

$$
\left\{\begin{array}{l}
T_{i t}^{o n} \geq T_{i \min }^{o n} \\
T_{i t}^{o f f} \geq T_{i \min }^{o f f}
\end{array}\right.
$$

Among them, the running time of each unit should be greater than its minimum value, and the closing time of each unit should also be greater than its minimum value.

\section{4) Reservoir capacity constraints}

Because the capacity of the water stored in the reservoir is limited; that is, at time t, E is used to characterize the capacity of the reservoir. This amount has two upper and lower limits, which is a constraint.

$$
E_{\min }^{i} \leq E_{t}^{i} \leq E_{\max }^{i}
$$

\section{5) Hydropower output constraints}

For hydropower stations, the discarding force is constrained to:

$$
P_{h t \text { min }} \leq P_{h t} \leq P_{h t \text { max }}
$$

$P_{h t}$ is the output of the hydropower station at time t; $P_{h t \text { min }}$ and $P_{h t \text { max }}$ are their minimum output and maximum output, respectively.

6) Constraints on overall output

Since the multi-energy complementary system has multiple energy components, the load required for the load is the sum of wind power, solar power, thermal power, and water and electricity:

$$
P_{l t}=\sum_{i=1}^{n} P_{i t}+P_{f t}+P_{s t}+P_{y t}
$$


Where $P_{l t}$ is the output required for the load at his time.

\section{C.Particle Swarm Optimization}

The basic principle of particle swarm optimization (PSO) is to initialize a group of particles without mass and volume and move in a certain region. Consider each solution of the problem to be solved as a particle, and use the fitness function to measure the particle's superiority. Inferior, and all particles always follow the current optimal particle, move in a certain speed and direction within the feasible solution region, and obtain the optimal solution of the problem after iterating multiple times ${ }^{[9]}$.

$$
\begin{gathered}
v_{i}^{d+1}=\omega v_{i}^{d}+c_{1} f_{\text {rand }}\left(p_{\text {best }, i}^{d}-x_{i}^{d}\right)+ \\
c_{2} f_{\text {rand }}\left(p_{\text {best }, i}^{d}-x_{i}^{d}\right)
\end{gathered}
$$

$x_{i}^{d+1}=x_{i}^{d}+\alpha v_{i}^{d+1}$

Where $\mathrm{i}$ is the number of particles; $\mathrm{d}$ is the number of iterations; $p_{\text {best }, i}^{d} 、 g_{\text {best }, i}^{d}$ is the individual optimal and global optimal of the ith particle in the dth iteration; $v_{i}^{d}, x_{i}^{d}$ is the velocity of the ith particle in the dth iteration Position; $\omega, c_{1}, c_{2}$ is the weight coefficient during the speed update process; $f_{\text {rand }}$ is a random number from 0 to 1 ; $\omega$ is the constraint factor, usually set to 1 . W uses the weight of linear variation ${ }^{[10]}$, the variation formula is:

$$
\omega=\omega_{\max }-\frac{t\left(\omega_{\max }-\omega_{\min }\right)}{t_{\max }}
$$

Where $\omega_{\min }$ and $\omega_{\max }$ are the minimum and maximum values of $\omega$, in general $\omega_{\min }=0.4, \omega_{\max }$
$=0.9 ; t_{\max }$ is the maximum number of iterations.

In this paper, clean energy, thermal power demand and grid load are used as particles, and each hour of 24 hours a day is the scheduling time. The algorithm flow is:

In this paper, clean energy, thermal power demand and grid load are used as particles, and each hour of 24 hours a day is the scheduling time. The algorithm flow is:

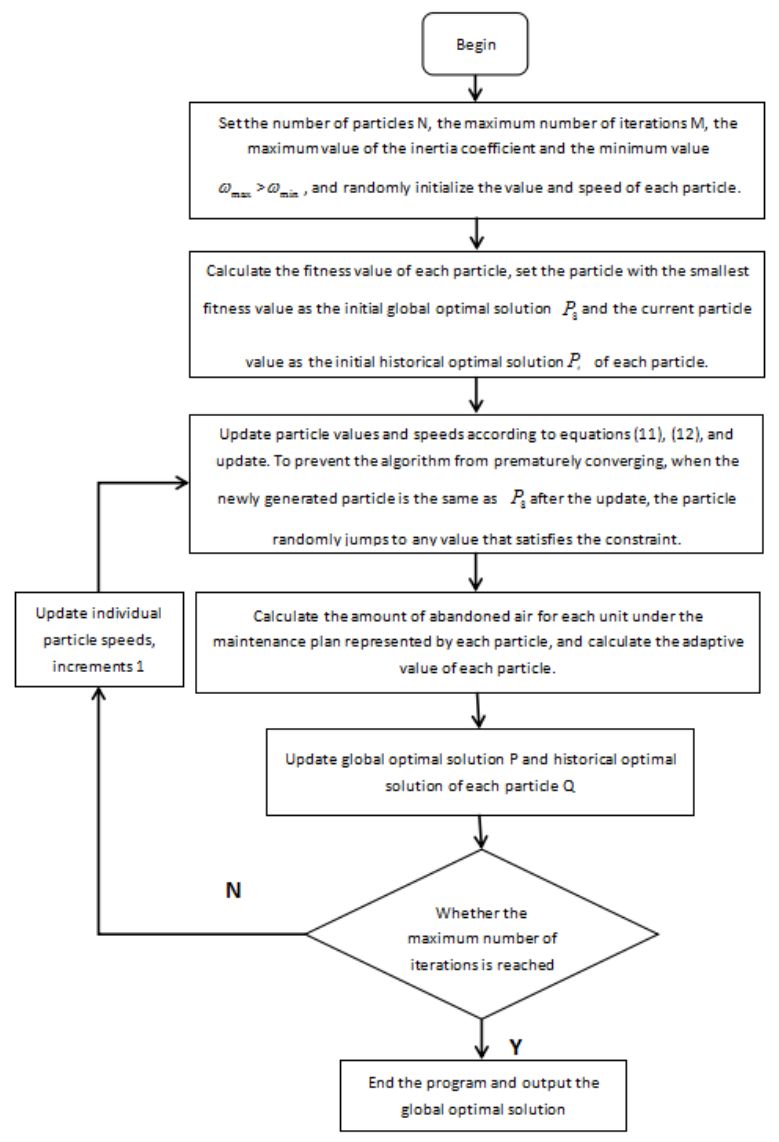

Fig.1 Algorithm flow chart

\section{V . Case analysis}

Take a certain place in Xinjiang as an example and analyze it according to the specific situation.Figure 1 below shows the design of multi-energy complementary structure in a certain area of Xinjiang. 


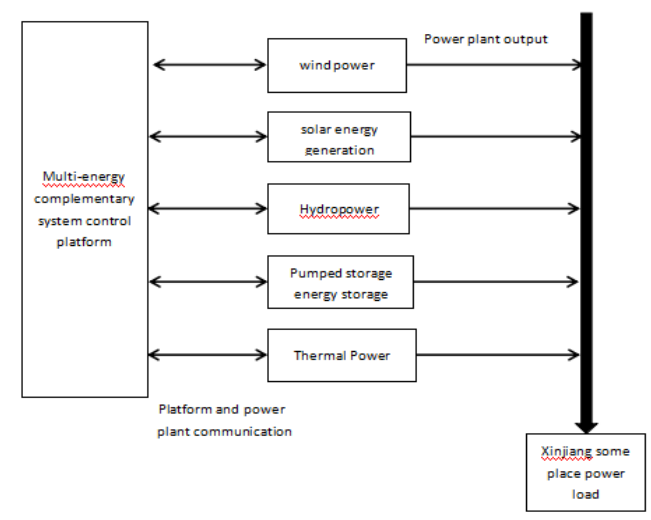

Fig. 1 Design drawings for multi energy complementary structures in Xinjiang

Some places in Xinjiang have two forms of new energy generation, namely wind power resources and solar power generation. The total installed capacity of the wind farm is $250 \mathrm{MW}$, and the photovoltaic power generation is about 50MW. Although there are no large hydropower plants in the area, there are certain energy storage facilities, and combined with hydropower resources in the surrounding areas, the total hydropower regulation capacity is about $500 \mathrm{MW}$, and the total thermal power generation capacity is about $1300 \mathrm{MW}$. In this paper, taking 24 hours a day as an example, the parameters are selected according to the operation situation of typical power stations of various energy forms in the area, multiplied by a certain proportion to simulate the operation of the whole area. After simulation, the calculation data is processed to obtain the demand for thermal power in the case of wind power output, wind and light complementation and wind and water complementation, as shown in Figures 2, 3 and 4.

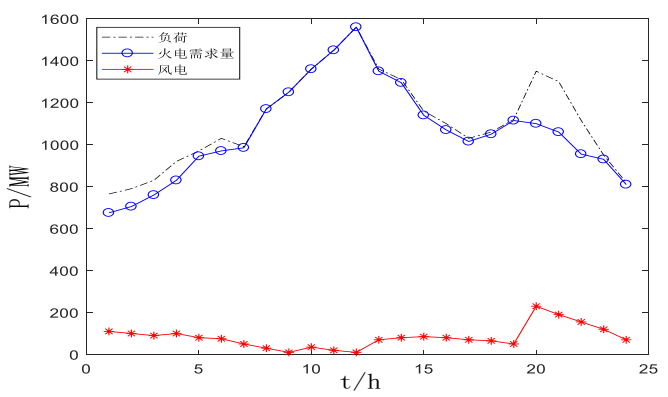

Fig.2 Thermal power demand of complementary system under wind power output

As can be seen from Figure 3 above, only wind power is input. It can be seen that in the case of only wind power input, the difference between the demand and the demand, that is, the power consumption required to supplement the conventional thermal power resources, is still in a state of very fluctuating. The reason for the analysis is mainly the instability of wind power, and the power of wind power generation changes during the day. In this case, due to the fact that the thermal power generation is not fast enough, and the huge loss caused by the opening and closing power, it cannot be combined with the wind power supply.

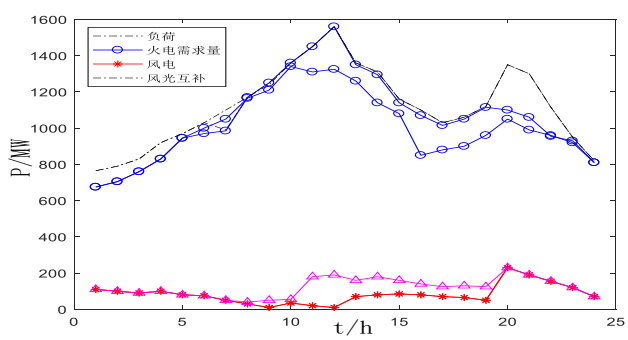

Fig.3 Thermal power demand of complementary systems under wind and solar energy

As can be seen from Figure 3 above, it can be seen that the fluctuation of the difference has been slightly reduced. This is because when the wind power fluctuates during the day, the solar power generation can perform a certain peak-shaping effect. If the scale of solar power generation is large enough, the effect will be better, but the solar input power parameters used in the simulation environment are relatively small, so the effect is not obvious. On the other hand, during the night hours, the suspension of solar power generation did not work. 


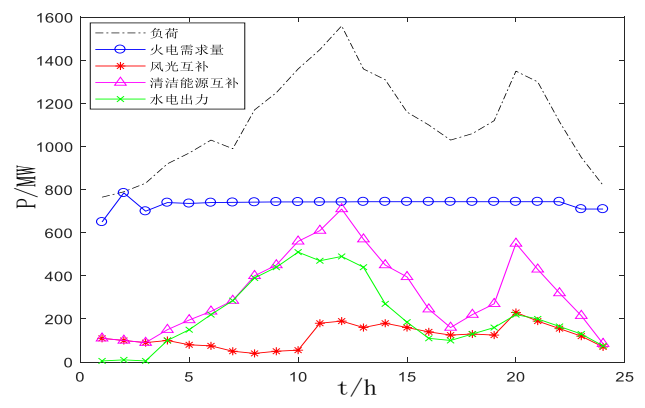

Fig.4 Thermal power demand by complementary systems under wind and solar, water energy

It can be obtained from the above figure 4, after the three multi-energy complementary structures of the scenery water are used. It can be seen that the difference between clean energy and total demand at this time is at a substantially constant and small value. This is because the regulation of hydropower and pumped storage, with the rapid adjustment of hydropower resources, is timely supplemented when wind power and solar power fluctuate. The all-weather adjustment of hydropower also compensates for the nighttime fluctuations of wind and light. In the case where the difference between the total demand and the clean energy is substantially constant, the remaining portion can be stably supplied by the thermal power. The consumption of thermal power resources is reduced, and thermal power does not require large-scale output capacity adjustment, which can save a lot of non-renewable energy.

Comprehensive modeling and simulation analysis shows that multi-energy complementarity is an effective means to improve the quality of wind power distribution and solve the problem of abandoned wind. Through the cooperation with solar energy, hydropower and other forms of clean energy, the value of new energy is fully exploited, and the use of traditional fossil energy is reduced, and the quality of the overall power supply is also guaranteed.

\section{Conclusion}

Multi-energy complementarity is a new energy dispatching strategy that calls various energy forms to cooperate with each other, complement each other, and foster strengths and weaknesses. By coordinating wind power resources with solar power resources, hydropower resources, and traditional thermal power resources, rational dispatching and coordinated output, it can effectively solve the problem of large wind power output volatility, not easy to be connected to the grid, and ensure the stability and power supply of the grid. Under the premise of stability, the use of clean new energy is fully utilized, reducing the use of fossil energy.

\section{Reference}

[1] Zhang Chuan, Yang Lei, Niu Tongyang, Zhang Mengyao.Comparison and Analysis of Energy Storage Techniques for Restraining Wind Power Output Fluctuation[J].Power System Protection and Control, 2015,43(07): 149-154.

[2] He Jianbo, $\mathrm{Hu}$ Zhijian, Liu Yukai.Establishment and solution of largescale multi-objective water -fire-wind coordinated optimal dispatching mode [J].Power System Protection and Control, 2015,43(06): 1-7.

[3] Liu Dan, Li Qiang, Yuan Xiaodong. A stochastic energy optimal dispatching model for microgrid [J].Power System Protection and Control, 2014, 42(11): 112-117.

[4] Ma Hongting, Zhang Chuanlong, song Shaw, Liang $\mathrm{Pu}$, Zhang Yufeng, dunna. Experimental research on the multi energy complementary heating system of solar energy source heat pump [J]. solar journal, 2014,35 (11): 21522158. 
[5] Ye Lin, Qu Xiaoxu, Yu Yanxiang, Zhang Jietan, Wang Yuefeng, Huang Yuehui, Wang Weisheng. Analysis of the operational characteristics of solar energy complementary power generation system within day time scale [J]. power system automation, 2018,42 (04): 158-164.

[6] Song Xuri, Ye Lin. Research on optimal allocation of wind / photovoltaic / diesel hybrid power generation system $[\mathrm{J}]$. power grid and clean energy, 2011,27 (05): 66-72.

[7] Ma Xiyuan, Guo Xiaobin, LeiJinyong.Distributed PV/Gas/Electric Hybrid Capacity Planning Method for Multipurpose Complementarity [J].Power System Automation, 2018,42(04): 55-63.

[8] Bai Kaifeng, Gu Jie, Peng Hongqiao, Zhu Binruo. The optimization of multi energy complementary microgrid systems generated by fusion of wind and scenery scenes: [J/OL]. power system automation: 1-9[2018-07-30].

[9] Ding Bojian, Zheng Xiuyu, Zhou Fengquan, Zhu Haojun, Ma Hongwei. Research on the capacity configuration method of multi energy complem- entary power supply in microgrid $[\mathrm{J}]$. power system protection and control, 2013,41 (16): 144-148

[10] Zu Qiwu, Niu Yugang, Chen Bei.Research on Multi-objective Economic Operation Strategy of Microgrid Based on Improved Particle Swarm Optimization[J].Power System Protection and Control, 2017,45(14): 57-63. 